\title{
Decentralized robust optimal control of large flexible space structures by local proper controllers using displacement output
}

\author{
Yohji KOBAYASHI* \\ *Department of Mechanical Engineering, Kobe City College of Technology \\ 8-3, Gakuen Higashi-machi, Nishi-ku, Kobe-shi, Hyogo 651-2194, Japan \\ E-mail: koba@kobe-kosen.ac.jp
}

Received 24 December 2014

\begin{abstract}
This paper considers position and attitude control of large flexible space structures composed of a number of subsystems(substructures) which are interconnected by flexible links modeled by springs and dampers. It is assumed that sensors and actuators are collocated in each subsystem. The purpose of the paper is to propose a decentralized control scheme by local proper controllers using only displacement output, which makes both each closed-loop subsystem and an overall closed-loop system not only robustly stable against uncertainty of characteristic parameters such as mass, damping, and stiffness, but also optimal for quadratic cost functions. First, we introduce a second order low-pass filter of which relative degree is 1 , at each input channel of each subsystem. Then, we temporarily feed back displacement and velocity output of the substructure and output of the filter to input of the filter locally, so that we obtain a local proper controller using displacement output. By choosing parameters of each local proper controller as it becomes a phase lead compensator, the closed-loop subsystems and the overall closed-loop system become robustly stable. Furthermore, it can be shown the closed-loop subsystems and the overall closed-loop system also become optimal for quadratic cost functions by making two feedback gains in each local proper controller sufficiently large. Finally, numerical examples are presented to show effectiveness of the proposed method.
\end{abstract}

Key words : Decentralized control, Large flexible space structure, Robust control, Optimal control, Proper controller, Displacement output

\section{Introduction}

This paper considers position and attitude control of large flexible space structures composed of a number of subsystems(substructures) which are interconnected by flexible links modeled by springs and dampers. Flexible interconnection of subsystems is realistic when we deal with very large space structures such as solar power satellites (Mankins, 1997). For interconnected large space structures, it is reasonable to apply decentralized control compatible with subsystems, which may allow us to expand a large space structure by connecting a new subsystem, or to remove a failed subsystem without any change of the control law. As such a control method, the direct velocity and displacement feedback (DVDFB) (Ikeda, Koujitani, and Kida, 1993) is useful under the assumption of sensors and actuators collocation, if the rigid modes are controllable and observable. It is easily seen that DVDFB can be implemented in a decentralized form (Kobayashi, Ikeda, Fujisaki, Matsuda, and Kida, 1999). Furthermore, DVDFB robustly stabilizes space structures against uncertainty of characteristic parameters such as mass, damping, and stiffness, and DVDFB makes an overall closed-loop system optimal for a quadratic cost function. However, for implementation of DVDFB, velocity/angular velocity sensors are needed added to displacement/angle sensors. (In the following pages, 'displacement' means 'displacement/angle'.) For reduction of costs, it is desired not to use such velocity/angular velocity sensors. To solve this problem, the dynamic displecement feedback (DDFB) has been proposed (Fujisaki, Ikeda, and Miki, 2001). DDFB adopts a proper dynamic controller using only displacement feedback, and also robustly stabilizes space structures with uncertain characteristic parameters and 
makes a closed-loop system optimal (Kobayashi, Ikeda, and Fujisaki, 2002) as well as DVDFB. However, DDFB has been proposed as a theoretical scheme (Fujisaki, Ikeda, and Miki, 2001), so that it has not been shown how to determine the parameters of a DDFB controller quantitatively. For this issue, it has been reported that local proper controllers using displacement output make each closed-loop subsystem and an overall closed-loop system robustly stable by choosing the local controller parameters as all the local controllers become phase lead compensators (Imoto and Kobayashi, 2012). The objective of this paper is to present a decentralized robust and optimal control method by local proper controllers using only displacement output for interconnected large flexible space structures with collocated sensors and actuators.

The basic idea is as follows. We first introduce a second order low-pass filter of which relative degree is 1 , at each input channel of each substructure. Then, we feed back temporarily displacement and velocity output of the substructure and output of the filter to input of the filter locally, so that we obtain a second order local proper controller using only displacement output. By choosing parameters of each local proper controller as it becomes a phase lead compensator, a closed-loop subsystem becomes robustly stable against uncertainty of characteristic parameters of each substructure such as mass, damping, and stiffness. Furthermore, by making two feedback gains in each local proper controller sufficiently large, we can realize both a wide frequency band of phase lead compensation and high gain of the controller, so that the closed-loop subsystem becomes optimal. After stabilizing each subsystem, we interconnect the closed-loop subsystems by flexible links modeled by springs and dampers, and it is shown the overall closed-loop system maintains robust stability and becomes optimal for a quadratic cost function under the condition that the local controller parameters make both a matrix given for the cost function of the overall system and a solution matrix of Riccati equation positive definite.

The organization of this paper is as follows. In Section 2, we describe subsystems of a large flexible space structure, and derive a second order local proper controller using only displacement output for each subsystem. Then, we show a closed-loop subsystem becomes robustly stable under the condition that the controller parameters make each local controller a phase lead compensator in Section 3, and we derive a condition for optimality of the closed-loop subsystems in Section 4. In Section 5, we describe the overall closed-loop system composed of substructures interconnected by flexible links, the decentralized low-pass filters, and the decentralized feedback. In Section 6, we show the local controllers with their parameters chosen in Section 3 and 4, make the overall closed-loop system robustly stable, and we derive another condition for optimality of the overall closed-loop system. Finally, numerical examples are presented to show effectiveness of the proposed method in Section 7.

\section{Space structure and local proper controller}

In this section, we consider a large flexible space structure composed of $\ell$ subsystems(substructures) with collocated sensors and actuators. In modeling the space structures precisely, they should be represented by infinite dimensional systems because they are essentially distributed parameter systems (Balas, 1982). However, it is difficult to design controllers for infinite dimensional systems, so that we practically represent flexible space structures as reduced order lumped parameter systems in designing control systems. We consider the $i$-th subsystem described by

$$
\begin{aligned}
& M_{i} \frac{d^{2} q_{i}(t)}{d t^{2}}+D_{i} \frac{d q_{i}(t)}{d t}+K_{i} q_{i}(t)=L_{i} u_{i}(t) \\
& y_{i}(t)=L_{i}^{T} q_{i}(t), \quad i=1,2, \ldots, \ell
\end{aligned}
$$

where $q_{i}(t) \in \mathfrak{R}^{n_{i}}, u_{i}(t) \in \mathfrak{R}^{r_{i}}$, and $y_{i}(t) \in \mathfrak{R}^{r_{i}}$ are respectively the displacement, control input, and measured output vectors of the $i$-th subsystem. The mass matrix $M_{i}$ is positive definite, and the damping and stiffness matrices $D_{i}, K_{i}$ are positive semi-definite because the existence of rigid modes implies

$$
\operatorname{rank}\left[\begin{array}{cc}
D_{i} & K_{i}
\end{array}\right]=\operatorname{rank} D_{i}=\operatorname{rank} K_{i}<n_{i}
$$

The input matrix $L_{i}$ is defined by the locations and directions of actuators, and the output matrix $L_{i}^{T}$ expresses the locations and directions of sensors. This transposition relation between the input and output matrices means the sensors/actuators collocation. We assume that

$$
\operatorname{rank}\left[\begin{array}{cc}
D_{i} & L_{i}
\end{array}\right]=n_{i}, \operatorname{rank}\left[\begin{array}{cc}
K_{i} & L_{i}
\end{array}\right]=n_{i}
$$

hold, which mean that the rigid modes of each subsystem are controllable and observable under the condition (3).

To control position and attitude of the $i$-th subsystem (1), (2), we first introduce the following second order low-pass filter of which relative degree is 1 , at each input channel of the subsystem.

$$
u_{i}(s)=\frac{\left(s+z_{i}\right)}{s\left(s+p_{i}\right)} v_{i}(s)
$$


where $p_{i}, z_{i}$ are positive scalars satisfying the following inequality

$$
z_{i}<p_{i}
$$

and $v_{i}(s)$ is input to the filter given by

$$
v_{i}(s)=-\gamma_{i}\left\{\alpha_{i} R_{i} y_{i}(s)+\beta_{i} R_{i} s y_{i}(s)+u_{i}(s)\right\}
$$

where $\alpha_{i}, \beta_{i}, \gamma_{i}$ are positive scalars, and $R_{i} \in \mathfrak{R}^{r_{i} \times r_{i}}$ is an arbitrary positive definite matrix.

By substituting Eq. (7) into Eq. (5), we obtain a local proper controller using displacement output $y_{i}(s)$ as follows.

$$
u_{i}(s)=-\frac{\gamma_{i} \beta_{i}\left(s+z_{i}\right)\left(s+\frac{\alpha_{i}}{\beta_{i}}\right)}{s^{2}+\left(p_{i}+\gamma_{i}\right) s+\gamma_{i} z_{i}} R_{i} y_{i}(s)=-\frac{\gamma_{i} \beta_{i}\left(s+z_{i}\right)\left(s+\frac{\alpha_{i}}{\beta_{i}}\right)}{\left(s+\tilde{p}_{i 1}\right)\left(s+\tilde{p}_{i 2}\right)} R_{i} y_{i}(s)
$$

where $-\tilde{p}_{i 1},-\tilde{p}_{i 2}\left(\tilde{p}_{i 1}<\tilde{p}_{i 2}\right)$ are two roots of the characteristic equation $s^{2}+\left(p_{i}+\gamma_{i}\right) s+\gamma_{i} z_{i}=0$ in Eq. (8).

\section{Robust stabilization of closed-loop subsystem}

In this section, we show that each local proper controller robustly stabilizes the closed-loop subsystem against uncertainty of characteristic parameters in each substructure. By choosing such the controller parameters in Eq. (8) as the proper controller becomes a phase lead compensator, the closed-loop subsystem becomes robustly stable against uncertain characteristic parameters of the substructure (Imoto and Kobayashi, 2012). It can be shown that the proper controller (8) becomes a phase lead compensator if Eq. (6) holds and if $\beta_{i}$ is sufficiently larger than $\alpha_{i}$ in the feedback (7). In fact, it is found that the relation

$$
\tilde{p}_{i 1}<z_{i}<\tilde{p}_{i 2},
$$

holds between the parameters of the controller (8) for $0<\gamma_{i}<\infty$ by checking root loci (Kuo, 1967) of the following transfer function

$$
\frac{s+z_{i}}{\left(s+\tilde{p}_{i 1}\right)\left(s+\tilde{p}_{i 2}\right)}
$$

which is obtained by eliminating $\gamma_{i} \beta_{i}\left(s+\frac{\alpha_{i}}{\beta_{i}}\right)$ from the numerator in the right side of Eq. (8). Here, it is possible to set $\alpha_{i} / \beta_{i}$ as $\alpha_{i} / \beta_{i}<\tilde{p}_{i 1}$ by choosing sufficiently larger $\beta_{i}$ than $\alpha_{i}$. As a result,

$$
\frac{\alpha_{i}}{\beta_{i}}<\tilde{p}_{i 1}<z_{i}<\tilde{p}_{i 2}
$$

holds as shown in Fig. 1. Then, the proper controller (8) becomes a phase lead compensator so that it makes the closedloop subsystem composed of the substructure (1), (2) and the controller (8) robustly stable against uncertain characteristic parameters such as mass, damping, and stiffness of the substructure.

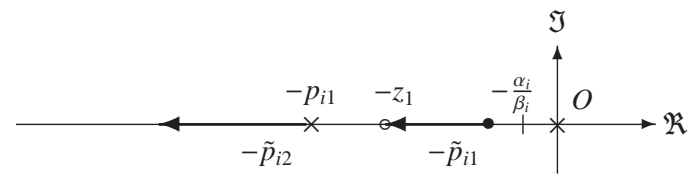

$\circ$ and $\times$ mean zeros and poles of low-pass filter (5).

Fig. 1 Loci of poles $\tilde{p}_{i 1}$ and $\tilde{p}_{i 2}$ as a function of $\gamma_{i}$

\section{Optimal control of closed-loop subsystem}

A realization of the low-pass filter (5) is described as follows.

$$
\begin{aligned}
& \frac{d}{d t}\left[\begin{array}{c}
\zeta_{i 1}(t) \\
\zeta_{i 2}(t)
\end{array}\right]=\left[\begin{array}{cc}
0 & I_{r_{i}} \\
0 & -p_{i} I_{r_{i}}
\end{array}\right]\left[\begin{array}{c}
\zeta_{i 1}(t) \\
\zeta_{i 2}(t)
\end{array}\right]+\left[\begin{array}{c}
0 \\
I_{r_{i}}
\end{array}\right] v_{i}(t) \\
& u_{i}(t)=\left[\begin{array}{ll}
z_{i} I_{r_{i}} & I_{r_{i}}
\end{array}\right]\left[\begin{array}{c}
\zeta_{i 1}(t) \\
\zeta_{i 2}(t)
\end{array}\right] \\
& \zeta_{i j}(t)=\left[\begin{array}{llll}
\zeta_{i j 1}(t) & \zeta_{i j 2}(t) & \cdots & \zeta_{i j r_{i}}(t)
\end{array}\right]^{T}, j=1,2 \\
& v_{i}(t)=\left[\begin{array}{llll}
v_{i 1}(t) & v_{i 2}(t) & \cdots & v_{i r_{i}}(t)
\end{array}\right]^{T}, u_{i}(t)=\left[\begin{array}{llll}
u_{i 1}(t) & u_{i 2}(t) & \cdots & u_{i r_{i}}(t)
\end{array}\right]^{T}
\end{aligned}
$$


where $I_{r_{i}} \in \mathfrak{R}^{r_{i} \times r_{i}}$ denotes an identity matrix of the $r_{i}$-th order.

We describe the augmented system composed of the substructure (1), (2) and the filter (12), (13) as follows.

$$
\begin{aligned}
\frac{d x_{i}(t)}{d t} & =A_{i} x_{i}(t)+B_{i} v_{i}(t) \\
x_{i}(t) & =\left[\begin{array}{c}
q_{i}(t) \\
d q_{i}(t) / d t \\
\zeta_{i 1}(t) \\
\zeta_{i 2}(t)
\end{array}\right], A_{i}=\left[\begin{array}{cccc}
0 & I_{n_{i}} & 0 & 0 \\
-M_{i}^{-1} K_{i} & -M_{i}^{-1} D_{i} & z_{i} M_{i}^{-1} L_{i} & M_{i}^{-1} L_{i} \\
0 & 0 & 0 & I_{r_{i}} \\
0 & 0 & 0 & -p_{i} I_{r_{i}}
\end{array}\right], B_{i}=\left[\begin{array}{c}
0 \\
0 \\
0 \\
I_{r_{i}}
\end{array}\right]
\end{aligned}
$$

where $I_{n_{i}} \in \mathfrak{R}^{n_{i} \times n_{i}}$ denotes an identity matrix of the $n_{i}$-th order.

Here, we should note that the closed-loop subsystem composed of the augmented system (14) and the control law (7) is equivalent to the substructure controlled by the local proper controller (8). Now, we present the following theorem for optimality of the closed-loop subsystems.

Theorem 1 For the $i$-th subsystem, the control law (7) minimizes the following quadratic cost function $J_{i}$, and the closed-loop subsystem becomes an optimal regulator,

$$
J_{i}=\int_{0}^{\infty}\left(x_{i}^{T}(t) \tilde{Q}_{i} x_{i}(t)+v_{i}^{T}(t) \tilde{R}_{i} v_{i}(t)\right) d t
$$

where matrices $\tilde{Q}_{i}$ and $\tilde{R}_{i}$ are respectively given as

$$
\begin{aligned}
& \tilde{Q}_{i}=T_{i}^{T} Q_{i} T_{i} \\
& T_{i}=\left[\begin{array}{cccc}
\gamma_{i} I_{n_{i}} & 0 & 0 & 0 \\
0 & \gamma_{i} I_{n_{i}} & 0 & 0 \\
0 & 0 & \gamma_{i} I_{r_{i}} & 0 \\
0 & 0 & 0 & I_{r_{i}}
\end{array}\right] \\
& Q_{i}=\left[\begin{array}{cccc}
2 \alpha_{i} K_{i}+\alpha_{i}^{2} L_{i} R_{i} L_{i}^{T} & 0 & 0 & \alpha_{i} p_{i} L_{i}+\beta_{i} K_{i} M_{i}^{-1} L_{i} \\
0 & 2\left(\beta_{i} D_{i}-\alpha_{i} M_{i}\right)+\beta_{i}^{2} L_{i} R_{i} L_{i}^{T} & 0 & \left(\beta_{i} p_{i}-\alpha_{i}\right) L_{i}+\beta_{i} D_{i} M_{i}^{-1} L_{i} \\
0 & 0 & z_{i}^{2} R_{i}^{-1} & 0 \\
\alpha_{i} p_{i} L_{i}^{T}+\beta_{i} L_{i}^{T} M_{i}^{-1} K_{i} & \left(\beta_{i} p_{i}-\alpha_{i}\right) L_{i}^{T}+\beta_{i} L_{i}^{T} M_{i}^{-1} D_{i} & 0 & \gamma_{i}\left\{2\left(p_{i}-z_{i}\right)+\gamma_{i}\right\} R_{i}^{-1}-2 \beta_{i} \gamma_{i} L_{i}^{T} M_{i}^{-1} L_{i}
\end{array}\right] \\
& \tilde{R}_{i}=R_{i}^{-1} \text {, }
\end{aligned}
$$

if the parameters $\alpha_{i}, \beta_{i}$, and $\gamma_{i}$ are chosen so as to make the matrix $\tilde{Q}_{i}$ and the following matrix $\tilde{P}_{i}$ positive definite.

$$
\begin{aligned}
\tilde{P}_{i}= & T_{i}^{T} P_{i} T_{i} \\
P_{i} & =\left[\begin{array}{cccc}
\alpha_{i} D_{i}+\beta_{i} K_{i}+\alpha_{i} \beta_{i} L_{i} R_{i} L_{i}^{T} & \alpha_{i} M_{i} & 0 & \alpha_{i} L_{i} \\
\alpha_{i} M_{i} & \beta_{i} M_{i} & 0 & \beta_{i} L_{i} \\
0 & 0 & z_{i}\left\{R_{i}^{-1}+\left(p_{i} R_{i}^{-1}-\beta_{i} L_{i}^{T} M_{i}^{-1} L_{i}\right) / \gamma_{i}\right\} & z_{i} R_{i}^{-1} \\
\alpha_{i} L_{i}^{T} & \beta_{i} L_{i}^{T} & z_{i} R_{i}^{-1} & \gamma_{i} R_{i}^{-1}
\end{array}\right]
\end{aligned}
$$

(Proof) Since unstable poles of the substructure (1), (2) are only rigid modes, that is, poles of $s=0$, the assumptions (4) imply that the substructure is stabilizable. In addition, there is no unstable pole-zero cancellation between the substructure and the low-pass filter (5), because the filter has no finite zero with a non-negative real part. Therefore, the augmented system (14) is stabilizable. When $\left(A_{i}, B_{i}\right)$ is a stabilizable pair, the following Riccati equation has the unique positive definite solution $\tilde{P}_{i}$ for the positive definite matrices $\tilde{Q}_{i}$ of Eq. (16) and $\tilde{R}_{i}$ of Eq. (17).

$$
\tilde{P}_{i} A_{i}+A_{i}^{T} \tilde{P}_{i}-\tilde{P}_{i} B_{i} \tilde{R}_{i}^{-1} B_{i}^{T} \tilde{P}_{i}=-\tilde{Q}_{i}
$$

It is easily seen that the matrix $\tilde{P}_{i}$ in Eq. (18) satisfies the Riccati equation (20). Furthermore, the matrix $\tilde{P}_{i}$ is positive definite by the condition of Theorem 1 . Then, the matrix $\tilde{P}_{i}$ is the unique positive definite solution of the Riccati equation (20). Using this $\tilde{P}_{i}$, the stabilizing feedback is calculated as

$$
v_{i}(t)=-\tilde{R}_{i}^{-1} B_{i}^{T} \tilde{P}_{i} x_{i}(t)=-\gamma_{i}\left\{\alpha_{i} R_{i} y_{i}(t)+\beta_{i} R_{i} \frac{d y_{i}(t)}{d t}+u_{i}(t)\right\} .
$$

This $v_{i}(t)$ corresponds to the feedback (7) so that the control law (7) makes the closed-loop subsystem optimal for the cost function (15). 
We should note that the existence of $\alpha_{i}, \beta_{i}$, and $\gamma_{i}$ is guaranteed because the matrix $\tilde{Q}_{i}$ and $\tilde{P}_{i}$ certainly become positive definite by choosing sufficiently larger $\beta_{i}$ than $\alpha_{i}$, and sufficiently large $\gamma_{i}$. On the other hand, as the condition (11) holds even if we make $\beta_{i}$ and $\gamma_{i}$ sufficiently large, the control law (7) makes the $i$-th closed-loop subsystem not only robustly stable but also optimal for the quadratic cost function (15).

This formulation of optimal control problems, in which a quadratic cost function is found for feedback stabilizing a closed-loop system, has been introduced as the inverse problem of optimal regulator(Fujii, 1988). In the inverse problem, a physical meaning of a quadratic cost function is not necessarily clear, however, the obtained feedback decreases sensitivity against parameter variation in a control object, and it suppresses amplitude of vibration of each state variable by evaluating state variables in the quadratic cost function. In the proposed method, the feedback (21) possesses these advantages in stabilizing the substructure (1), (2).

(Remark) Examples of gain and phase diagrams of a local proper controller are shown in Fig. 2, in which solid lines indicate the gain and phase diagrams for large $\beta_{i}$ and $\gamma_{i}$, and broken lines indicate those for small $\beta_{i}$ and $\gamma_{i}$. From Fig. 2, it is found that a lower boundary of a phase lead compensation interval decreases by choosing sufficiently large $\beta_{i}$ to $\alpha_{i}$, and also note that a upper boundary of the interval and gain of the proper controller increase by choosing large $\gamma_{i}$. Optimality of the closed-loop subsystem is achieved by the wider frequency band width of phase lead compensation and the higher gain of the local proper controller.
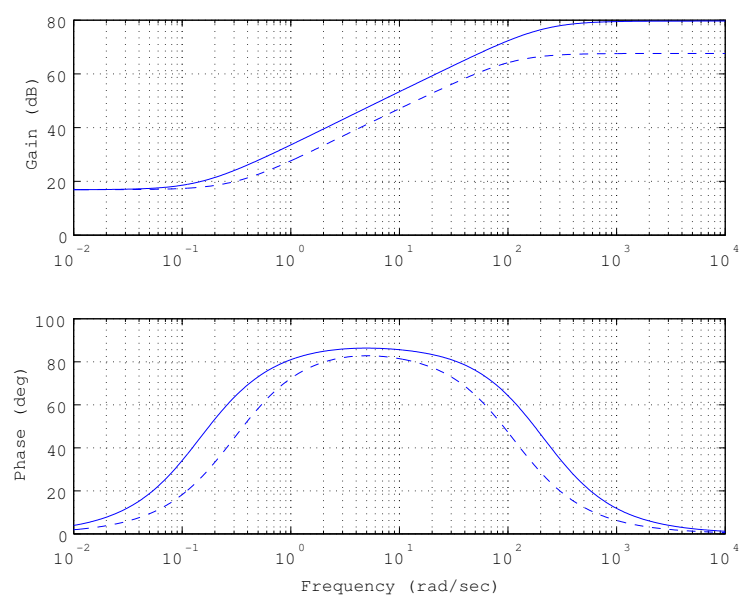

Fig. 2 Example of Bode diagram of local proper controller

\section{Overall closed-loop system}

Here, we represent an overall closed-loop system. First, the $i$-th substructure with interconnection to other substructures is described by

$$
\begin{aligned}
M_{i} \frac{d^{2} q_{i}(t)}{d t^{2}}+D_{i} \frac{d q_{i}(t)}{d t}+K_{i} q_{i}(t)=L_{i} u_{i}(t) & +\sum_{j=1}^{\ell} N_{i j}\left[K_{C i j}\left(N_{j i}^{T} q_{j}(t)-N_{i j}^{T} q_{i}(t)\right)\right. \\
& \left.+D_{C i j}\left(N_{j i}^{T} \frac{d q_{j}(t)}{d t}-N_{i j}^{T} \frac{d q_{i}(t)}{d t}\right)\right], i=1,2, \ldots, \ell
\end{aligned}
$$

where $N_{i j}$ is a matrix representing the locations and directions of flexible links modeled by springs and dampers at the $i$-th subsystem, which connect the $i$-th subsystem with the $j$-th subsystem. The matrices $K_{C i j}$ and $D_{C i j}$ respectively denote spring and damper parameters, and we assume that they are positive definite.

The term in the right side of Eq. (22)

$$
K_{C i j}\left(N_{j i}^{T} q_{j}(t)-N_{i j}^{T} q_{i}(t)\right)+D_{C i j}\left(N_{j i}^{T} \frac{d q_{j}(t)}{d t}-N_{i j}^{T} \frac{d q_{i}(t)}{d t}\right)
$$

represents force and/or torque affecting the $i$-th subsystem by the springs and dampers connected with the $j$-th subsystem, and $N_{i j}^{T} q_{i}$ is the displacement of the connecting points of springs and dampers in the $i$-th subsystem. The above description has been obtained under the assumption that when the $i$-th and $j$-th subsystems are at the origins of their 
displacements/angles, that is, $q_{i}=0$ and $q_{j}=0$, the springs between them are neither stretched nor compressed. Since the effects of springs and dampers are bilateral, $K_{C i j}=K_{C j i}$ and $D_{C i j}=D_{C j i}$ hold.

Then, an overall closed-loop system is composed of an overall augmented system, that is, interconnected substructures with local low-pass filters, and decentralized feedback. The interconnected substructures are described by

$$
\begin{aligned}
& \bar{M} \frac{d^{2} \bar{q}(t)}{d t^{2}}+\bar{D} \frac{d \bar{q}(t)}{d t}+\bar{K} \bar{q}(t)=\bar{L} \bar{u}(t) \\
& \bar{y}(t)=\bar{L}^{T} \bar{q}(t),
\end{aligned}
$$

where vectors and matrices are represented as follows.

$$
\begin{aligned}
& \bar{q}(t)=\left[\begin{array}{llll}
q_{1}^{T}(t) & q_{2}^{T}(t) & \cdots & q_{\ell}^{T}(t)
\end{array}\right]^{T}, \bar{u}(t)=\left[\begin{array}{lllll}
u_{1}^{T}(t) & u_{2}^{T}(t) & \cdots & u_{\ell}^{T}(t)
\end{array}\right]^{T}, \bar{y}(t)=\left[\begin{array}{llll}
y_{1}^{T}(t) & y_{2}^{T}(t) & \cdots & y_{\ell}^{T}(t)
\end{array}\right]^{T} \\
& \bar{M}=\operatorname{diag}\left\{M_{i}\right\}_{i=1,2 \ldots, \ell}, \bar{D}=\operatorname{diag}\left\{D_{i}\right\}_{i=1,2, \ldots, \ell}+\sum_{i=1}^{\ell-1} \sum_{j=i+1}^{\ell} \bar{N}_{i j} D_{C i j} \bar{N}_{i j}^{T}, \bar{K}=\operatorname{diag}\left\{K_{i}\right\}_{i=1,2, \ldots, \ell}+\sum_{i=1}^{\ell-1} \sum_{j=i+1}^{\ell} \bar{N}_{i j} K_{C i j} \bar{N}_{i j}^{T} \\
& \bar{L}=\operatorname{diag}\left\{L_{i}\right\}_{i=1,2 \ldots, \ell}
\end{aligned}
$$

In the matrix $\bar{N}_{i j}$, the elements except the submatrices $N_{i j}$ of the $i$-th block and $-N_{j i}$ of the $j$-th block, are all zero.

$$
\begin{aligned}
& \bar{N}_{i j}=\left[\begin{array}{lllll}
\cdots & N_{i j}^{T} & \cdots & -N_{j i}^{T} & \cdots
\end{array}\right] \\
& i \quad j
\end{aligned}
$$

The local low-pass filters are written by

$$
\begin{aligned}
& \frac{d}{d t}\left[\begin{array}{l}
\bar{\zeta}_{1}(t) \\
\bar{\zeta}_{2}(t)
\end{array}\right]=\left[\begin{array}{cc}
0 & I_{\bar{r}} \\
0 & -\bar{p} I_{\bar{r}}
\end{array}\right]\left[\begin{array}{l}
\bar{\zeta}_{1}(t) \\
\bar{\zeta}_{2}(t)
\end{array}\right]+\left[\begin{array}{c}
0 \\
I_{\bar{r}}
\end{array}\right] \bar{v}(t) \\
& \bar{u}(t)=\left[\begin{array}{ll}
\bar{z} I_{\bar{r}} & I_{\bar{r}}
\end{array}\right]\left[\begin{array}{l}
\bar{\zeta}_{1}(t) \\
\bar{\zeta}_{2}(t)
\end{array}\right] \\
& \bar{\zeta}_{1}(t)=\left[\begin{array}{llll}
\zeta_{11}^{T}(t) & \zeta_{21}^{T}(t) & \cdots & \zeta_{\ell 1}^{T}(t)
\end{array}\right]^{T}, \bar{\zeta}_{2}(t)=\left[\begin{array}{llll}
\zeta_{12}^{T}(t) & \zeta_{22}^{T}(t) & \cdots & \zeta_{\ell 2}^{T}(t)
\end{array}\right]^{T} \\
& \bar{v}(t)=\left[\begin{array}{cccc}
v_{1}^{T}(t) & v_{2}^{T}(t) & \cdots & v_{\ell}^{T}(t)
\end{array}\right]^{T}, \bar{p}=\operatorname{diag}\left\{p_{i} I_{r_{i}}\right\}_{i=1,2 \ldots, \ell}, \bar{z}=\operatorname{diag}\left\{z_{i} I_{r_{i}}\right\}_{i=1,2 \ldots, \ell}, \bar{r}=\sum_{i=1}^{\ell} r_{i}
\end{aligned}
$$

where $I_{\bar{r}} \in \mathfrak{R}^{\bar{r} \times \bar{r}}$ denotes an identity matrix of the $\bar{r}$-th order.

Finally, the decentralized feedback is given as follows.

$$
\begin{aligned}
\bar{v}(t) & =-\bar{\gamma}\left\{\bar{\alpha} \hat{R} \bar{y}(t)+\bar{\beta} \hat{R} \frac{d \bar{y}(t)}{d t}+\bar{u}(t)\right\} \\
\bar{\alpha} & =\operatorname{diag}\left\{\alpha_{i} I_{r_{i}}\right\}_{i=1,2 \ldots, \ell}, \bar{\beta}=\operatorname{diag}\left\{\beta_{i} I_{r_{i}}\right\}_{i=1,2 \ldots, \ell}, \bar{\gamma}=\operatorname{diag}\left\{\gamma_{i} I_{r_{i}}\right\}_{i=1,2 \ldots, \ell}, \hat{R}=\operatorname{diag}\left\{R_{i}\right\}_{i=1,2 \ldots, \ell}
\end{aligned}
$$

The overall augmented system is obtained by connecting the substructures (23), (24) and the low-pass filters (25), (26). The state equation of the overall augmented system is represented by

$$
\begin{aligned}
\frac{d \bar{x}(t)}{d t} & =\bar{A} \bar{x}(t)+\bar{B} \bar{v}(t) \\
\bar{x}(t) & =\left[\begin{array}{c}
\bar{q}(t) \\
d \bar{q}(t) / d t \\
\bar{\zeta}_{1}(t) \\
\bar{\zeta}_{2}(t)
\end{array}\right], \bar{A}=\left[\begin{array}{cccc}
0 & I_{\bar{n}} & 0 & 0 \\
-\bar{M}^{-1} \bar{K} & -\bar{M}^{-1} \bar{D} & \bar{M}^{-1} \bar{L} \bar{z} & \bar{M}^{-1} \bar{L} \\
0 & 0 & 0 & I_{\bar{r}} \\
0 & 0 & 0 & -\bar{p} I_{\bar{r}}
\end{array}\right], \bar{B}=\left[\begin{array}{c}
0 \\
0 \\
0 \\
I_{\bar{r}}
\end{array}\right] \\
\bar{n} & =\sum_{i=1}^{\ell} n_{i},
\end{aligned}
$$

where $I_{\bar{n}} \in \mathfrak{R}^{\bar{n} \times \bar{n}}$ denotes an identity matrix of the $\bar{n}$-th order.

We should note that the overall augmented system (28) controlled by the feedback (27) is equivalent to the overall closed-loop system interconnecting the closed-loop subsystems controlled by the local proper controllers (8). 


\section{Robust stabilization and optimal control of overall closed-loop system}

It has been reported that the overall closed-loop system controlled by local proper controllers using displacement output, is robustly stable if parameters of each local controller are chosen to satisfy inequalities (11) (Imoto and Kobayashi, 2012), and the parameters of each local proper controller (8) are satisfied with inequalities (11) even if we make $\beta_{i}$ and $\gamma_{i}$ sufficiently large. From these facts, it is concluded that the overall closed-loop system obtained in Section 5 also becomes robustly stable.

Now, we present the following theorem for optimality of the overall closed-loop system.

Theorem 2 The control law (27) minimizes the following quadratic cost function, and the overall closed-loop system becomes an optimal regulator,

$$
\bar{J}=\int_{0}^{\infty}\left(\bar{x}^{T}(t) \bar{Q} \bar{x}(t)+\bar{v}^{T}(t) \bar{R} \bar{v}(t)\right) d t
$$

where matrices $\bar{Q}$ and $\bar{R}$ are given as

$$
\begin{array}{cc}
\bar{Q}=\left[\begin{array}{cc}
\hat{\gamma}^{2} \hat{\alpha} K+K \hat{\alpha} \hat{\gamma}^{2}+\hat{\gamma}^{2} \hat{\alpha}^{2} \bar{L} \bar{R} \bar{L}^{T} & \frac{1}{2}\left(\hat{\gamma}^{2} \hat{\alpha} \bar{D}-\bar{D} \hat{\alpha} \hat{\gamma}^{2}\right)+\frac{1}{2}\left(\bar{K} \hat{\beta} \hat{\gamma}^{2}-\hat{\gamma}^{2} \hat{\beta} \bar{K}\right) \\
\frac{1}{2}\left(\bar{D} \hat{\alpha} \hat{\gamma}^{2}-\hat{\gamma}^{2} \hat{\alpha} \bar{D}\right)+\frac{1}{2}\left(\hat{\gamma}^{2} \hat{\beta} \bar{K}-\bar{K} \hat{\beta} \hat{\gamma}^{2}\right) & \hat{\gamma}^{2} \hat{\beta} \bar{D}+\bar{D} \hat{\beta} \hat{\gamma}^{2}-\left(\hat{\gamma}^{2} \hat{\alpha} \bar{M}+\bar{M} \hat{\alpha} \hat{\gamma}^{2}\right)+\hat{\gamma}^{2} \hat{\beta}^{2} \bar{L} \bar{R} \bar{L}^{T} \\
0 & 0 \\
\bar{p} \bar{L}^{T} \hat{\alpha} \hat{\gamma}+\bar{L}^{T} \hat{\beta} \hat{\gamma} \bar{M}^{-1} \bar{K} & \bar{p} \bar{L}^{T} \hat{\beta} \hat{\gamma}-\bar{L}^{T} \hat{\alpha} \hat{\gamma}+\bar{L}^{T} \hat{\beta} \hat{\gamma} \bar{M}^{-1} \bar{D} \\
0 & \hat{\gamma} \hat{\alpha} \bar{L} \bar{p}+\bar{K} \bar{M}^{-1} \hat{\gamma} \hat{\beta} \bar{L} \\
0 & 0 \\
\bar{\gamma}^{2} \bar{z}^{2} \bar{R}^{-1} & \\
0 & \bar{\gamma}(\bar{p}-\bar{z}) \bar{R}^{-1}+\bar{R}^{-1}(\bar{p}-\bar{z}) \bar{\gamma}+\bar{\gamma}^{2} \bar{R}^{-1}-\bar{L}^{T} \hat{\beta} \hat{\gamma} \bar{M}^{-1} \bar{L}-\bar{L}^{T} \bar{M}^{-1} \hat{\gamma} \hat{\beta} \bar{L}
\end{array}\right] \\
\bar{R}=\hat{R}^{-1} \quad \\
\hat{\alpha}=\operatorname{diag}\left\{\alpha_{i} I_{n_{i}}\right\}_{i=1,2 \ldots, \ell}, \hat{\beta}=\operatorname{diag}\left\{\beta_{i} I_{n_{i}}\right\}_{i=1,2 \ldots, \ell}, \hat{\gamma}=\operatorname{diag}\left\{\gamma_{i} I_{n_{i}}\right\}_{i=1,2 \ldots, \ell},
\end{array}
$$

if the parameters $\alpha_{i}, \beta_{i}$, and $\gamma_{i}$ for all subsystems are chosen so as to make the matrix $\bar{Q}$ and the following matrix $\bar{P}$ positive definite.

$$
\bar{P}=\left[\begin{array}{ccc}
\frac{1}{2}\left(\hat{\gamma}^{2} \hat{\alpha} \bar{D}+\bar{D} \hat{\alpha} \hat{\gamma}^{2}\right)+\frac{1}{2}\left(\hat{\gamma}^{2} \hat{\beta} \bar{K}+\bar{K} \hat{\beta} \hat{\gamma}^{2}\right)+\hat{\gamma}^{2} \hat{\alpha} \hat{\beta} \bar{L} \bar{R} \bar{L}^{T} & \hat{\gamma}^{2} \hat{\alpha} \bar{M} & \\
\bar{M} \hat{\alpha} \hat{\gamma}^{2} & \hat{\gamma}^{2} \hat{\beta} \bar{M} & \\
0 & 0 & \\
\bar{L}^{T} \hat{\alpha} \hat{\gamma} & \bar{L}^{T} \hat{\beta} \hat{\gamma} & \hat{\gamma} \hat{\alpha} \bar{L} \\
& 0 & \hat{\gamma} \hat{\beta} \bar{L} \\
\bar{\gamma}^{2} \bar{z} \bar{R}^{-1}+\bar{\gamma} \bar{z} \bar{R}^{-1}-\bar{L}^{T} \bar{M}^{-1} \hat{z} \hat{\beta} \bar{L} & \bar{\gamma} \bar{z} \bar{R}^{-1} \\
\bar{R}^{-1} \bar{z} \bar{\gamma} & \bar{\gamma} \bar{R}^{-1}
\end{array}\right]
$$

where $\hat{z}=\operatorname{diag}\left\{z_{i} I_{n_{i}}\right\}_{i=1,2 \ldots, \ell}$.

(Proof) Since the assumptions in Eq. (4) imply

$$
\operatorname{rank}\left[\begin{array}{cc}
\bar{D} & \bar{L}
\end{array}\right]=\bar{n}, \operatorname{rank}\left[\begin{array}{cc}
\bar{K} & \bar{L}
\end{array}\right]=\bar{n},
$$

the large space structure (23), (24) is stabilizable. In addition, there is no unstable pole-zero cancellation between the structure (23), (24) and the local low-pass filters (25), (26), because the filters have no zero at the origin. Therefore, the augmented system (28) is stabilizable.

When $(\bar{A}, \bar{B})$ is a stabilizable pair, the following Riccati equation

$$
\bar{P} \bar{A}+\bar{A}^{T} \bar{P}-\bar{P} \bar{B} \bar{R}^{-1} \bar{B}^{T} \bar{P}+\bar{Q}=0
$$

has the unique positive definite solution $\bar{P}$ for the positive definite matrices $\bar{Q}$ of (30) and $\bar{R}$ of (31).

It is easily seen that the matrix $\bar{P}$ in Eq. (32) satisfies the Riccati equation (34). Furthermore, $\bar{P}$ is positive definite by the condition of Theorem 2. Therefore, $\bar{P}$ is the unique positive definite solution of the Riccati equation (34). The following feedback using this $\bar{P}$, stabilizes the augmented system (28), and minimizes the cost function (29).

$$
\bar{v}(t)=-\bar{R}^{-1} \bar{B}^{T} \bar{P} \bar{x}(t)==-\bar{\gamma}\left\{\hat{\alpha} \hat{R} \bar{y}(t)+\bar{\beta} \hat{R} \frac{d \bar{y}(t)}{d t}+\bar{u}(t)\right\}
$$


This feedback $\bar{v}(t)$ corresponds to Eq. (27). Thus, the control law (27) stabilizes the augmented system (28) and makes the overall closed-loop system optimal for the quadratic cost function (29). The proof is completed.

(Remark) The existence of the feedback gains in Eq. (27) which satisfy the condition of Theorem 2 is also guaranteed. For example, we can choose the same values of $\alpha_{i}, \beta_{i}$, and $\gamma_{i}$ for all subsystems as

$$
\alpha_{i}=\alpha, \beta_{i}=\beta, \gamma_{i}=\gamma, \text { for all } i
$$

Then, the condition of Theorem 2 is satisfied by choosing sufficiently larger $\beta$ than $\alpha$, and sufficiently large $\gamma$. However, it is obvious that we can choose other gains which satisfy the condition of Theorem 2 in different ways.

\section{Example}

\subsection{Space structure and local controllers}

Let us consider an interconnected space structure, which is composed of two substructures connected in the $x$ direction with flexible links modeled by springs and dampers as illustrated in Fig. 3. This is a very simple model of a solar power satellite(Mankins, 1997), in which rigid bodies such as solar cell arrays are interconnected by metal tubes. Actually, the rigid bodies are so large that we can approximate characteristics of the metal tubes supporting the rigid bodies as those of flexible links modeled by springs and dampers. For simplicity, we assume that each substructure consists of two rigid bodies of rectangular shapes of the same size, which are connected in the $y$ direction by flexible links. The mass and moment of inertia of the $j$-th rigid body $(j=1,2)$ in the $i$-th substructure $(i=1,2)$ are denoted by $m_{i j}$ and $J_{i j}$. The motion of the rigid body is described by the displacements $x_{i j}, y_{i j}$ of the center of mass and the rotational angle $\theta_{i j}$ around the center of mass. It is assumed that the input forces and torques are applied at the centers of mass 11 and mass 22.

In the $i$-th substructure, each rigid body has two connecting points labeled as $i j k$ meaning that the connecting point is the $k$-th one $(k=1,2)$ at the $j$-th rigid body. The length of the line segment between the center of mass and the connecting point $i j k$ is denoted by $\ell_{i j k}$. The angle between the line segment and the edge of the rigid body at the connecting point $i j k$ is denoted by $\psi_{i j k}$. The springs and dampers are represented by lines between rigid bodies in Fig. 3. The angle between the edge of the rigid body and the direction of the spring and damper attached not rectangularly at the point $i j k$ is denoted by $\phi_{i j k}$. Then, the displacement vector of the $i$-th substructure in Eq. (1) is represented by

$$
q_{i}=\left[\begin{array}{llllll}
x_{i 1} & y_{i 1} & \theta_{i 1} & x_{i 2} & y_{i 2} & \theta_{i 2}
\end{array}\right]^{T}, i=1,2
$$

and the coefficient matrices in Eq. (1) are as follows.

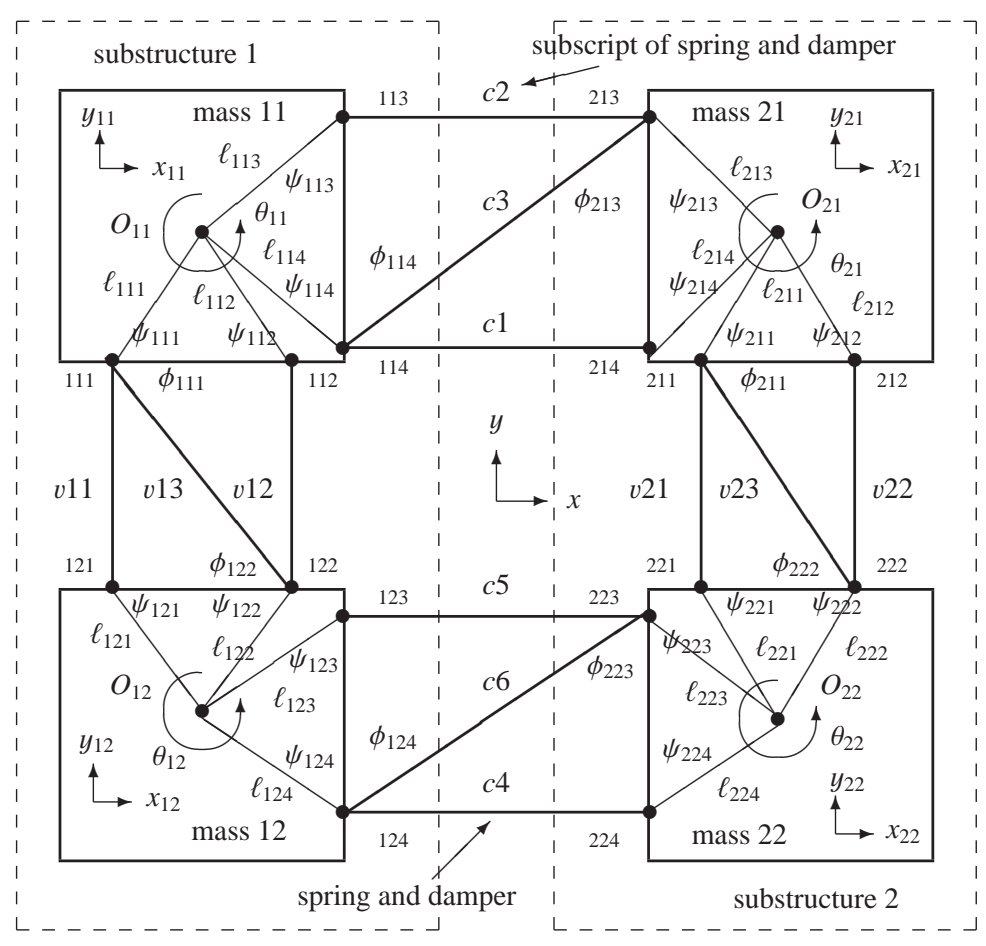

Fig. 3 Space structure 


$$
\begin{aligned}
& M_{i}=\operatorname{diag}\left\{m_{i 1}, m_{i 1}, J_{i 1}, m_{i 2}, m_{i 2}, J_{i 2}\right\} \\
& D_{i}=\left[\begin{array}{c}
\hat{N}_{i 1} \\
-\hat{N}_{i 2}
\end{array}\right] D_{s i}\left[\begin{array}{ll}
\hat{N}_{i 1}^{T} & -\hat{N}_{i 2}^{T}
\end{array}\right], \quad K_{i}=\left[\begin{array}{c}
\hat{N}_{i 1} \\
-\hat{N}_{i 2}
\end{array}\right] K_{s i}\left[\begin{array}{cc}
\hat{N}_{i 1}^{T} & -\hat{N}_{i 2}^{T}
\end{array}\right] \\
& \hat{N}_{i 1}=\left[\begin{array}{ccc}
0 & 0 & -\cos \phi_{i 11} \\
1 & 1 & \sin \phi_{i 11} \\
-\ell_{i 11} \cos \psi_{i 11}, & \ell_{i 12} \cos \psi_{i 12}, & -\ell_{i 11} \sin \left(\psi_{i 11}+\phi_{i 11}\right)
\end{array}\right] \\
& \hat{N}_{i 2}=\left[\begin{array}{ccc}
0 & 0 & -\cos \phi_{i 22} \\
1 & 1 & \sin \phi_{i 22} \\
\ell_{i 21} \cos \psi_{i 21}, & -\ell_{i 22} \cos \psi_{i 22}, & -\ell_{i 22} \sin \left(\psi_{i 22}+\phi_{i 22}\right)
\end{array}\right] \\
& D_{s i}=\operatorname{diag}\left\{d_{v i 1}, d_{v i 2}, d_{v i 3}\right\}, K_{s i}=\operatorname{diag}\left\{k_{v i 1}, k_{v i 2}, k_{v i 3}\right\} \\
& L_{1}=\left[\begin{array}{ll}
I_{3} & 0_{3}
\end{array}\right]^{T}, \quad L_{2}=\left[\begin{array}{ll}
0_{3} & I_{3}
\end{array}\right]^{T}
\end{aligned}
$$

Here, the matrices $\hat{N}_{i 1}$ and $\hat{N}_{i 2}$ are defined by the location and direction of springs and dampers in the $i$-th substructure. The scalars $k_{v i l}, d_{v i l}(l=1,2,3)$ respectively represent the spring and damper parameters. The subscript vil indicates the position of the spring and damper as in Fig. 3.

The substructures are connected at four points labeled as $i j k$ denoting the $k$-th point $(k=3,4)$ at the $j$-th rigid body $(j=1,2)$ in the $i$-th substructure $(i=1,2)$. Then, the interconnection term in Eq. (22) is expressed by

$$
\begin{aligned}
& \tilde{N}_{12}=\left[\begin{array}{lll}
N_{12}^{T} & -N_{21}^{T}
\end{array}\right]^{T} \\
& N_{12}=\operatorname{diag}\left\{\left[\begin{array}{ccc}
1 & 1 & \sin \phi_{1 j 4} \\
0 & 0 & \cos \phi_{1 j 4} \\
\ell_{1 j 4} \cos \psi_{1 j 4} & -\ell_{1 j 3} \cos \psi_{1 j 3} & \ell_{1 j 4} \sin \left(\psi_{1 j 4}+\phi_{1 j 4}\right)
\end{array}\right]\right\}_{j=1,2} \\
& N_{21}=\operatorname{diag}\left\{\left[\begin{array}{ccc}
1 & 1 & \sin \phi_{2 j 3} \\
0 & 0 & \cos \phi_{2 j 3} \\
-\ell_{2 j 4} \cos \psi_{2 j 4} & \ell_{2 j 3} \cos \psi_{2 j 3} & \ell_{2 j 3} \sin \left(\psi_{2 j 3}+\phi_{2 j 3}\right)
\end{array}\right]\right\}_{j=1,2} \\
& D_{C 12}=\operatorname{diag}\left\{d_{c 1}, d_{c 2}, d_{c 3}, d_{c 4}, d_{c 5}, d_{c 6}\right\}, K_{C 12}=\operatorname{diag}\left\{k_{c 1}, k_{c 2}, k_{c 3}, k_{c 4}, k_{c 5}, k_{c 6}\right\} .
\end{aligned}
$$

The scalars $k_{c p}$ and $d_{c p}(p=1, \ldots, 6)$ respectively represent the parameters of the springs and dampers connecting the substructures. The subscript $p$ indicates the $p$-th connection as shown in Fig. 3 . The notations $\ell_{i j k}, \psi_{i j k}, \phi_{i j k}$ for $k=3,4$ are defined as those for $k=1,2$.

For simulation, we use the nominal parameters of mass, moment of inertia, stiffness, and damping in each substructure as Case 1 shown in Table 1. To show the proposed controller robustly stabilizes the overall closed-loop system, we present another set of the structure parameters as Case 2, which contains perturbed characteristic parameters in mass, moment of inertia, stiffness, and damping as shown in Table 2. The length $\ell_{i j k}$ and angles $\psi_{i j k}(i, j=1,2, k=1,2,3,4)$ are respectively 1.0 , and 60 (deg). The angles $\phi_{111}, \phi_{122}, \phi_{211}, \phi_{222}$ are $60(\mathrm{deg})$.

In interconnections, we use the values of stiffness and damping

$$
k_{c p}=100.0, d_{c p}=1.0, p=1, \ldots, 6,
$$

and angles $\phi_{114}, \phi_{124}, \phi_{213}, \phi_{223}$ are given as $70(\mathrm{deg})$.

For the structure with the nominal characteristic parameters in Table 1, the local controller parameters $z_{i}, p_{i}, \alpha_{i}, \beta_{i}$, and $\gamma_{i}(i=1,2)$ are chosen as in Table 3 . The matrices $R_{i}(i=1,2)$ are respectively $3 \times 3$ identity matrices. These parameters are chosen so that the controller 1 becomes a phase lead compensator for natural resonance angular frequencies of each substructure. It is seen these parameters satisfy both the condition (11) for robust stabilization and the condition of Theorem 2, that is, these parameters make $\bar{Q}$ and $\bar{P}$ positive definite.

Table 1 Case 1: Nominal parameters of space structure

\begin{tabular}{c|c|c|c|c|c}
\hline$i$ & $j$ & $m_{i j}$ & $J_{i j}$ & $k_{v i l}$ & $d_{v i l}$ \\
\hline \multirow{2}{*}{1} & 1 & 10.0 & 5.0 & & \\
\cline { 2 - 4 } & 2 & 10.0 & 5.0 & 800 & 0.9 \\
\hline \multirow{2}{*}{2} & 1 & 10.0 & 5.0 & & \\
\cline { 2 - 4 } & 2 & 50.0 & 25.0 & & \\
\hline \multicolumn{5}{c}{} & \multicolumn{5}{c}{$l=1,2,3$}
\end{tabular}

Table 2 Case 2: Perturbed parameters of space structure

\begin{tabular}{c|c|c|c|c|c}
\hline$i$ & $j$ & $m_{i j}$ & $J_{i j}$ & $k_{v i l}$ & $d_{v i l}$ \\
\hline \multirow{2}{*}{1} & 1 & 15.0 & 7.5 & & \\
\cline { 2 - 4 } & 2 & 15.0 & 7.5 & \multirow{4}{*}{400} & 0.45 \\
\hline \multirow{2}{*}{2} & 1 & 15.0 & 5.0 & & \\
\cline { 2 - 4 } & 2 & 75.0 & 37.5 & & \\
\hline \multicolumn{5}{c}{$l=1,2,3$}
\end{tabular}


We can choose the other parameters of local controllers as shown in Table 4, which satisfy the condition (11), so that they make the overall closed-loop system robustly stable. However, these parameters do not satisfy the conditions of Theorem 2, because $\beta_{i}$ and $\gamma_{i}$ are so small that the matrix $\bar{Q}$ in (30) is not positive definite.

Table 3 Parameters of local controllers for robust optimal control(controller 1 )

\begin{tabular}{c|c|c|c|c|c}
\hline$i$ & $z_{i}$ & $p_{i}$ & $\alpha_{i}$ & $\beta_{i}$ & $\gamma_{i}$ \\
\hline 1 & 3.0 & 60.0 & 1.5 & 15.0 & 800.0 \\
\hline 2 & 1.0 & 30.0 & 2.0 & 20.0 & 693.0 \\
\hline
\end{tabular}

Table 4 Parameters of local controllers for robust control(controller 2)

\begin{tabular}{c|c|c|c|c|c}
\hline$i$ & $z_{i}$ & $p_{i}$ & $\alpha_{i}$ & $\beta_{i}$ & $\gamma_{i}$ \\
\hline 1 & 3.0 & 60.0 & 1.5 & 10.0 & 22.0 \\
\hline 2 & 1.0 & 30.0 & 2.0 & 12.0 & 20.0 \\
\hline
\end{tabular}
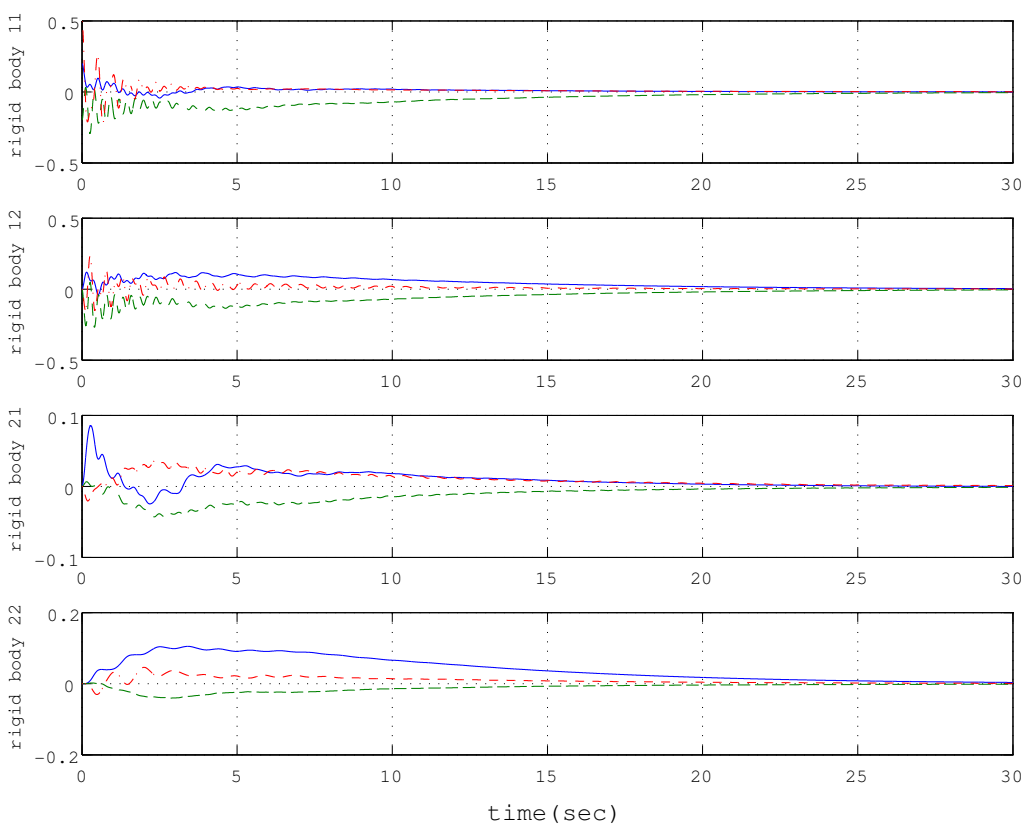

(a) Closed-loop system with controller 1
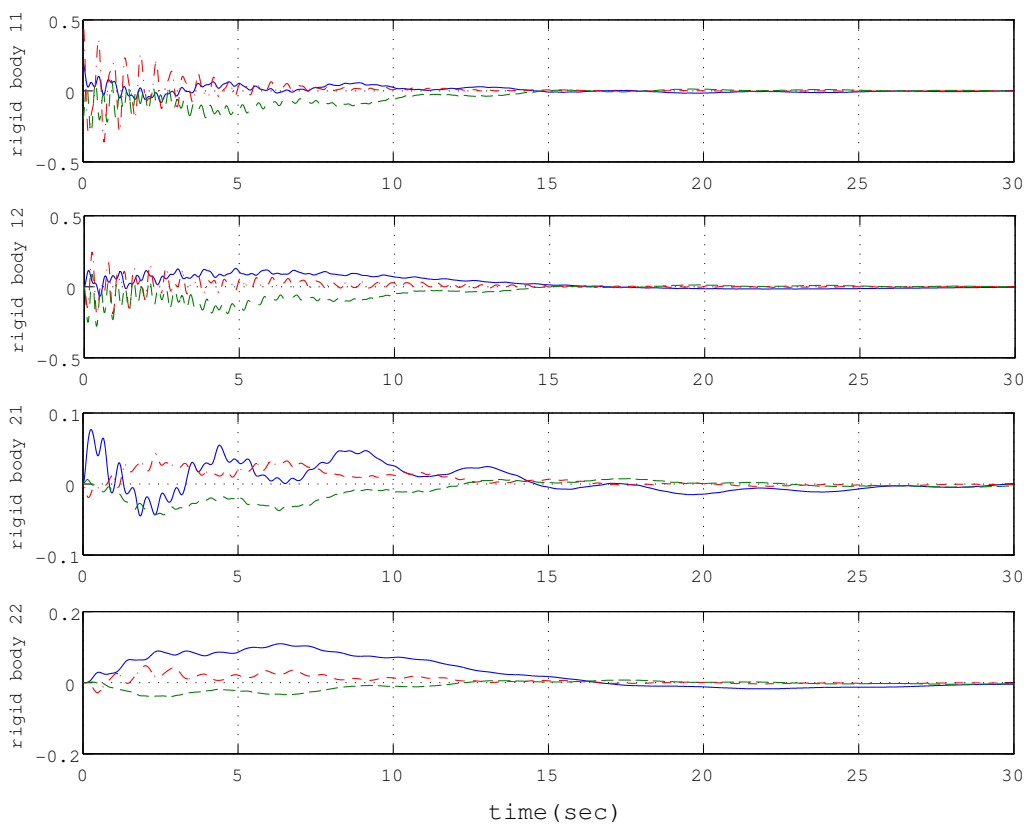

(b) Closed-loop system with controller 2

Fig. 4 Initial-state responses of displacements and rotational angles 


\subsection{Optimal control}

Using the characteristic parameters of the structure in Case 1, and setting initial displacements and rotation angles of the structure as

$$
x_{11}(0)=0.2, y_{11}(0)=-0.2, \theta_{11}(0)=0.5
$$

and other initial displacements and rotation angles are all 0 . The initial state responses of the overall closed-loop system using controller 1 in Table 3 are computed as in Fig. 4 (a), and those of controller 2 in Table 4 are in Fig. 4 (b), respectively.

The top, second, third, and bottom figures respectively show the responses of rigid bodies $11,12,21$, and 22 . Solid lines, broken lines, and chained lines respectively indicate the displacements in the direction $x_{i j}, y_{i j}$, and the rotation angle $\theta_{i j}$. It is found that the settling time and amplitude of vibration are less in Fig. 4 (a) than in Fig. 4 (b).

\subsection{Robust stabilization}

To evaluate the performance of robust stabilization by the proposed method, we calculate the initial state responses of the overall closed-loop system conposed of the structure with parameters of Case 2 in Table 2, and controller 1 in Table 3. The responses are obtained as shown in Fig. 5. The initial displacements and rotation angles of the structure, the figure arrangement(top, second, third, and bottom figures), and meaning of lines(solid lines, broken lines, and chained lines) are also the same as the examples in 7.2.

From these figures, it is seen that controller 1 with the parameters in Table 3 also stabilizes the overall closed-loop system with the parameters of Case 2. It shows that the overall closed-loop system becomes robustly stable against uncertain charactreristic parameters of the structure by the proposed method.
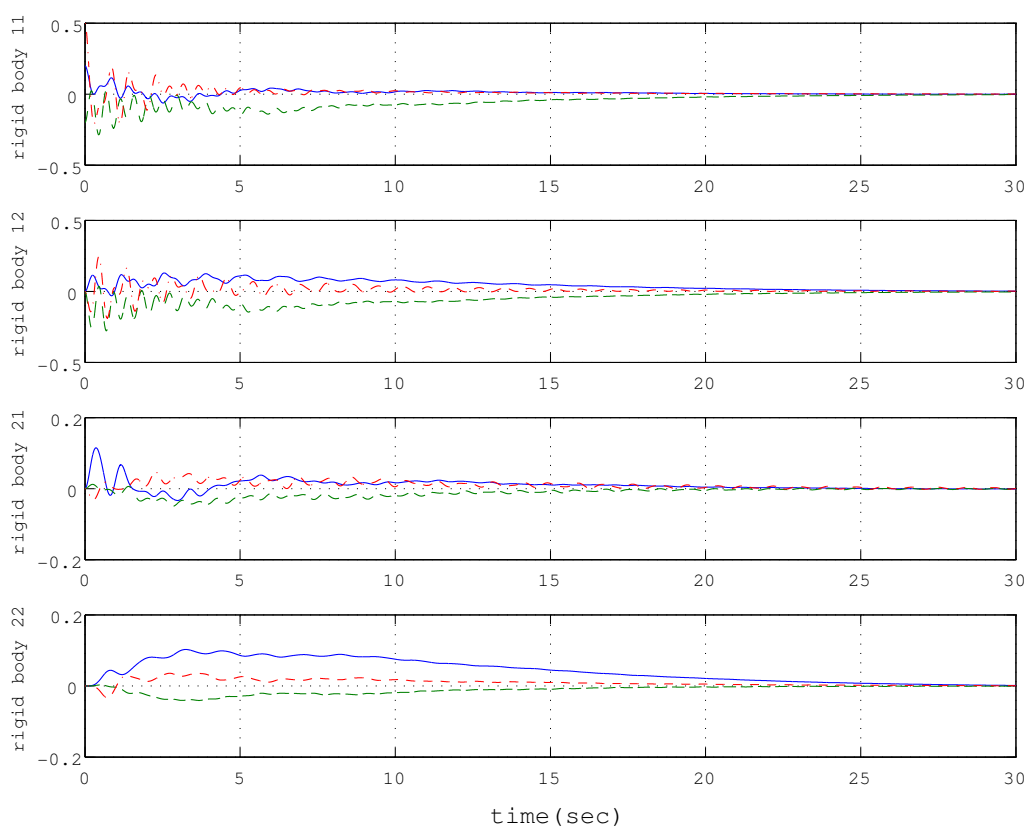

Fig. 5 Initial-state responses of displacements and rotational angles in closed-loop system with parameters of Case 2

\section{Conclusion}

This paper has considered position and attitude control of large flexible space structures composed of a number of substructures which are connected by flexible links modeled by springs and dampers. A decentralized control method by local proper controlllers using only displacement output has been proposed under the assumption that sensors and actuators are collocated.

We have shown the local proper controllers robustly stabilize the closed-loop subsystems if the parameters of each local controller are chosen as it becomes a phase lead compensator, and we have derived the condition of the controller parameters which make the closed-loop subsystem optimal, in which two feedback gains $\beta_{i}$ and $\gamma_{i}$ are chosen sufficiently large. Furthermore, we have shown the local proper controllers also robustly stabilize the overall closed-loop system, 
and we have derived the condition of the controller parameters which make the overall closed-loop system optimal for a quadratic cost function. The overall closed-system becomes optimal if two feedback gains $\beta_{i}$ and $\gamma_{i}$ in all subsystems are chosen sufficiently large, so that both the matrix $\bar{Q}$ in the quadratic cost function and the matrix $\bar{P}$ of the solution of Riccati equation become positive definite. These results can be used in choosing the controller parameters on the design stage and also in tuning them on the operation stage.

\section{References}

Balas, M. J., Trends in Large Space Structure Control Theory: Fondest Hopes, Wildest Dreams, IEEE Transactions on Automatic Control, Vol.27, No.3 (1982), pp.522-535.

Fujii, T., The Inverse Problem of Optimal Regulator, Journal of the Society of Instrument and Control Engineers, Vol. 27, No. 8 (1988), pp.717-726 (in Japanese).

Fujisaki, Y., Ikeda, M., and Miki, K., Robust Stabilization of Large Space Structures Via Displacement Feedback, IEEE Transactions on Automatic Control, Vol.46, No.12 (2001), pp.1993-1996.

Ikeda, M., Koujitani, K., and Kida, T., Optimality of Direct Velocity and Displacement Feedback for Large Space Structures with Collocated Sensors and Actuators, Preprints of the 12th IFAC World Congress, Vol.VI (1993), pp.91-94.

Imoto, R. and Kobayashi, Y., Decentralized Control for Flexible Large Space Structures by Local Proper Controllers, Preprints of the 55th Japan Joint Automatic Control Conference (2012), pp.935-938 (In Japanese).

Kobayashi, Y., Ikeda, M., and Fujisaki, Y., Optimal Control of Large Space Structures by Simple Dynamic Displacement Feedback, Transactions of the Society of Instrument and Control Engineers, Vol.38, No.8 (2002), pp.694-701 (in Japanese).

Kobayashi, Y., Ikeda, M., Fujisaki, Y., Matsuda, M., and Kida, T., Decentralized DVDFB Control of Large Space Structures: Fault Tolerance and Optimality, Transactions of the Society of Instrument and Control Engineers, Vol.35, No.7 (1999), pp.919-925 (in Japanese).

Kuo, B. C., Automatic Control Systems (1967), pp.329-388, Prentice Hall, Englewood Cliffs, NJ.

Mankins, J. C., The Space Solar Power Option, Aerospace America, Vol.35 (1997), pp.30-36. 